\title{
A Systematic Approach for Model-Based Aircraft Engine Performance Estimation
}

Donald L. Simon and Sanjay Garg

Glenn Research Center, Cleveland, Ohio 


\section{NASA STI Program . . . in Profile}

Since its founding, NASA has been dedicated to the advancement of aeronautics and space science. The NASA Scientific and Technical Information (STI) program plays a key part in helping NASA maintain this important role.

The NASA STI Program operates under the auspices of the Agency Chief Information Officer. It collects, organizes, provides for archiving, and disseminates NASA's STI. The NASA STI program provides access to the NASA Aeronautics and Space Database and its public interface, the NASA Technical Reports Server, thus providing one of the largest collections of aeronautical and space science STI in the world. Results are published in both non-NASA channels and by NASA in the NASA STI Report Series, which includes the following report types:

- TECHNICAL PUBLICATION. Reports of completed research or a major significant phase of research that present the results of NASA programs and include extensive data or theoretical analysis. Includes compilations of significant scientific and technical data and information deemed to be of continuing reference value. NASA counterpart of peer-reviewed formal professional papers but has less stringent limitations on manuscript length and extent of graphic presentations.

- TECHNICAL MEMORANDUM. Scientific and technical findings that are preliminary or of specialized interest, e.g., quick release reports, working papers, and bibliographies that contain minimal annotation. Does not contain extensive analysis.

- CONTRACTOR REPORT. Scientific and technical findings by NASA-sponsored contractors and grantees.
- CONFERENCE PUBLICATION. Collected papers from scientific and technical conferences, symposia, seminars, or other meetings sponsored or cosponsored by NASA.

- SPECIAL PUBLICATION. Scientific, technical, or historical information from NASA programs, projects, and missions, often concerned with subjects having substantial public interest.

- TECHNICAL TRANSLATION. Englishlanguage translations of foreign scientific and technical material pertinent to NASA's mission.

Specialized services also include creating custom thesauri, building customized databases, organizing and publishing research results.

For more information about the NASA STI program, see the following:

- Access the NASA STI program home page at http://www.sti.nasa.gov

- E-mail your question via the Internet to help@ sti.nasa.gov

- Fax your question to the NASA STI Help Desk at 443-757-5803

- Telephone the NASA STI Help Desk at 443-757-5802

- Write to: NASA Center for AeroSpace Information (CASI) 7115 Standard Drive Hanover, MD 21076-1320 
NASA/TM—2010-216077

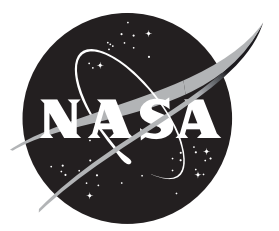

\section{A Systematic Approach for Model-Based Aircraft Engine Performance Estimation}

Donald L. Simon and Sanjay Garg

Glenn Research Center, Cleveland, Ohio

Prepared for the

Infotech@Aerospace (I@A) Conference

sponsored by the American Institute of Aeronautics and Astronautics

Seattle, Washington, April 6-9, 2009

National Aeronautics and

Space Administration

Glenn Research Center

Cleveland, Ohio 44135 


\section{Acknowledgments}

This research was conducted under the NASA Aviation Safety Program, Integrated Vehicle Health Management Project.

The authors graciously acknowledge Jonathan Litt for his support and insightful discussions which led to the development of this work.

Trade names and trademarks are used in this report for identification only. Their usage does not constitute an official endorsement, either expressed or implied, by the National Aeronautics and Space Administration.

Level of Review: This material has been technically reviewed by technical management.

Available from

NASA Center for Aerospace Information 7115 Standard Drive

Hanover, MD 21076-1320
National Technical Information Service 5285 Port Royal Road Springfield, VA 22161 


\title{
A Systematic Approach for Model-Based Aircraft Engine Performance Estimation
}

\author{
Donald L. Simon and Sanjay Garg \\ National Aeronautics and Space Administration \\ Glenn Research Center \\ Cleveland, Ohio 44135
}

\begin{abstract}
A requirement for effective aircraft engine performance estimation is the ability to account for engine degradation, generally described in terms of unmeasurable health parameters such as efficiencies and flow capacities related to each major engine module. This paper presents a linear point design methodology for minimizing the degradation- induced error in model-based aircraft engine performance estimation applications. The technique specifically focuses on the underdetermined estimation problem, where there are more unknown health parameters than available sensor measurements. A condition for Kalman filterbased estimation is that the number of health parameters estimated cannot exceed the number of sensed measurements. In this paper, the estimated health parameter vector will be replaced by a reduced order tuner vector whose dimension is equivalent to the sensed measurement vector. The reduced order tuner vector is systematically selected to minimize the theoretical mean squared estimation error of a maximum a posteriori estimator formulation. This paper derives theoretical estimation errors at steady-state operating conditions, and presents the tuner selection routine applied to minimize these values. Results from the application of the technique to an aircraft engine simulation are presented and compared to the estimation accuracy achieved through conventional maximum a posteriori and Kalman filter estimation approaches. Maximum a posteriori estimation results demonstrate that reduced order tuning parameter vectors can be found that approximate the accuracy of estimating all health parameters directly. Kalman filter estimation results based on the same reduced order tuning parameter vectors demonstrate that significantly improved estimation accuracy can be achieved over the conventional approach of selecting a subset of health parameters to serve as the tuner vector. However, additional development is necessary to fully extend the methodology to Kalman filter-based estimation applications.
\end{abstract}

\section{Nomenclature}

$\begin{array}{ll}A, A_{x h}, A_{x q}, & \\ B, B_{x h}, B_{x q}, & \\ C, C_{x h}, C_{x q}, & \text { system matrices } \\ D, F, F_{x h}, F_{x q}, & \\ G, L, M, N & \\ \text { C-MAPSS } & \text { Commercial Modular Aero-Propulsion System Simulation } \\ \text { Fn } & \text { net thrust } \\ G_{h}, G_{z} & \text { estimation bias matrices } \\ H & \text { matrix that relates health parameter effects to measured steady-state engine outputs } \\ H_{q} & \text { matrix that relates tuner vector effects to measured steady-state engine outputs } \\ H_{z} & \text { matrix that relates health parameter effects to unmeasured steady-state engine outputs } \\ \text { HPC } & \text { high pressure compressor } \\ \text { HPT } & \text { high pressure turbine } \\ I & \text { identity matrix } \\ K_{\infty} & \text { Kalman filter gain }\end{array}$




$\begin{array}{ll}\text { LPC } & \text { low pressure compressor } \\ \text { LPT } & \text { low pressure turbine } \\ \text { MAP } & \text { maximum a posteriori } \\ P_{h}, P_{z} & \text { health and auxiliary parameter covariance matrices } \\ P_{\hat{h}, k}, P_{\hat{z}, k} & \text { covariance matrices of estimated parameters } \\ P_{\infty} & \text { Kalman filter state estimation covariance matrix } \\ Q, Q_{x h}, Q_{x q} & \text { process noise covariance matrices } \\ R & \text { measurement noise covariance matrix } \\ \text { SmLPC } & \text { LPC stall margin } \\ \text { T40 } & \text { combustor exit temperature } \\ \text { T50 } & \text { LPT exit temperature } \\ V^{*} & \text { transformation matrix relating } h \text { to } q \\ h & \text { health parameter vector } \\ q & \text { reduced order tuner vector } \\ u_{k} & \text { actuator command vector } \\ v_{k} & \text { measurement noise vector } \\ w_{k}, w_{h, k}, w_{x h, k} & \text { process noise vectors } \\ x_{k} & \text { state vector } \\ x_{x h, k} & \text { augmented state vector, }\left[x_{k}^{T} h_{k}^{T}\right]^{T} \\ x_{x q, k} & \text { reduced order augmented state vector, }\left[x_{k}^{T} q_{k}^{T}\right]^{T} \\ y_{k} & \text { vector of measured outputs } \\ z_{k} & \text { vector of unmeasured (auxiliary) outputs } \\ \varepsilon_{k} & \text { residual vector (estimate minus its expected value) } \\ \eta & \text { efficiency health parameter } \\ \gamma & \text { flow capacity health parameter } \\ & \end{array}$

\section{Subscripts}

$k \quad$ discrete time step index

$x h \quad$ augmented state vector $(x$ and $h)$

$x q \quad$ reduced order state vector $(x$ and $q)$

ss steady-state value

\section{Superscripts}

$\begin{array}{ll}\dagger & \text { pseudoinverse } \\ \wedge & \text { estimated value } \\ \sim & \text { error value } \\ - & \text { mean value } \\ \mathrm{T} & \text { transpose }\end{array}$

\section{Operators}

$E[\cdot] \quad$ expected value of argument

$\operatorname{tr}\{\cdot\} \quad$ trace of matrix

$\operatorname{SSEE}(\cdot) \quad$ sum of squared estimation errors 


\section{Introduction}

An emerging approach in the field of aircraft engine controls and health management is the inclusion of real-time on-board models for the in-flight estimation of engine performance variations (Refs. 1,2, and3). This technology, typically based on Kalman filter concepts, enables the estimation of unmeasured engine performance parameters which can be directly utilized by controls, prognostics and health management applications. A challenge that complicates this practice is the fact that an aircraft engine's performance is affected by its level of degradation, generally described in terms of unmeasurable health parameters such as efficiencies and flow capacities related to each major engine module. Through Kalman filter-based estimation techniques, the level of engine performance degradation can be estimated, given that there are at least as many sensors as parameters to be estimated (Ref. 4). However, in an aircraft engine the number of sensors available is typically less than the number of health parameters, presenting an underdetermined estimation problem. A common approach to address this shortcoming is to estimate a subset of the health parameters, referred to as model tuning parameters. While this approach enables online Kalman filter-based estimation, it can result in "smearing" the effects of unestimated health parameters onto those that are estimated, and in turn reduces the accuracy of model-based estimation schemes. Recently, Litt (Ref. 5) presented an approach based on singular value decomposition that selects a model tuning parameter vector of low enough dimension to be estimated by a Kalman filter. The model tuning parameter vector, $q$, is constructed as a linear combination of all health parameters, $h$, given by

$$
q=V^{*} h
$$

where the transformation matrix, $V^{*}$, is selected by applying singular value decomposition to capture the overall effect of the larger set of health parameters on the engine variables as closely as possible in the least squares sense. In this paper a new linear point design technique that applies a systematic approach to tuning parameter selection will be presented. This technique, like the one presented in Reference 5, defines a transformation matrix, $V^{*}$, used to construct a reduced order tuning parameter vector that is a linear combination of all health parameters, and of low enough dimension to enable Kalman filter estimation. The new approach selects a reduced order tuner vector, $q$, that minimizes the theoretical mean squared estimation error produced when applying maximum a posteriori (MAP) estimation. MAP estimation, sometimes referred to as regularization, is commonly applied for ground-based aircraft turbine engine gas path analysis (Refs. 6 and 7). It is applied to "snap shot" engine measurements collected at quasi-steady-state operating points during each flight, and takes advantage of prior knowledge of engine health parameter distributions. Unlike the Kalman filter, it is not a recursive estimator and it does not account for system dynamics, therefore the MAP estimator is not well suited for dynamic parameter estimation applications like in-flight aircraft engine performance estimation. However, the MAP estimator is capable of estimating more unknowns than available measurements due to its inclusion of $a$ priori knowledge of the estimated parameters' covariance. In this study, the MAP estimator formulation has been chosen to facilitate reduced order tuner parameter selection for two reasons. First, its theoretical estimation errors can be more readily derived than those of the Kalman filter. Second, the MAP estimation accuracy achieved through reduced order tuner estimation can be directly compared to the MAP estimation accuracy achieved by estimating the entire health parameter vector. The ability of the parameter estimation accuracy achieved through reduced order tuner estimation to approximate that of full order health parameter estimation will verify that a $V^{*}$ transformation matrix optimal for MAP estimation has indeed been produced. There is no known closed form solution for selecting $V^{*}$ to satisfy the objective of minimizing the theoretical MAP estimation error. Therefore, a multivariable iterative search routine is applied to perform this function. Once $V^{*}$ and the reduced order $q$ vector are produced, they can be applied to model-based aircraft engine performance estimation applications based on either MAP estimation or Kalman filter estimation. 
The remaining sections of this paper are organized as follows. First, linear dynamic state space equations and steady-state measurement process equations for the engine are introduced, for both full order (health parameter vector) and reduced order (tuner parameter vector) formulations. The application of these equations to formulate the Kalman filter and the MAP estimator equations is then presented. This is followed by a derivation of the MAP estimator theoretical mean squared estimation error. The theoretical MAP estimation error information is directly used by the iterative search routine applied to optimally select the reduced order tuning parameter vector, which is described next. Example MAP and Kalman filter estimation results from the application of the new methodology to an aircraft turbofan engine simulation are then shown. After the example results, there is a discussion of findings and required future work. Finally, conclusions are presented.

\section{Problem Formulation}

\section{A. Dynamic State Space Equations}

This subsection will first present the full order dynamic state space equations representative of engine dynamics around a linear design point, followed by a presentation of the reduced order dynamic state space equations. The latter can be applied to formulate the Kalman filter estimator.

\section{Full Order Dynamic State Space Equations}

The discrete linear time invariant engine state space equations about a linear design point are given as

$$
\begin{aligned}
x_{k+1} & =A x_{k}+B u_{k}+L h_{k}+w_{k} \\
y_{k} & =C x_{k}+D u_{k}+M h_{k}+v_{k} \\
z_{k} & =F x_{k}+G u_{k}+N h_{k}
\end{aligned}
$$

where $k$ is the time index, $x$ is the vector of state variables, $u$ is the vector of control inputs, $y$ is the vector of measured outputs, and $z$ is the vector of auxiliary (unmeasured) model outputs. The vector $h$ represents the engine health parameters, which induce shifts in other variables as the health parameters deviate from their nominal values. The vectors $w$ and $v$ are uncorrelated zero mean white noise input sequences. $Q$ will be used to denote the covariance of $w$, and $R$ to denote the covariance of $v$. The matrices $A, B, C, D, F, G$, $L, M$, and $N$ are of appropriate dimension. The health parameters, represented by the vector $h$, are unknown inputs to the system. They may be treated as a set of biases, and are thus modeled without dynamics. With this interpretation, Equation (2) can be written as:

$$
\begin{aligned}
& {\left[\begin{array}{c}
x_{k+1} \\
h_{k+1}
\end{array}\right]=\underbrace{\left[\begin{array}{cc}
A & L \\
0 & I
\end{array}\right]}_{A_{x h}} \underbrace{\left[\begin{array}{c}
x_{k} \\
h_{k}
\end{array}\right]}_{x_{x h, k}}+\underbrace{\left[\begin{array}{c}
B \\
0
\end{array}\right]}_{B_{x h}} u_{k}+\underbrace{\left[\begin{array}{c}
w_{k} \\
w_{h, k}
\end{array}\right]}_{w_{x h, k}}=A_{x h} x_{x h, k}+B_{x h} u_{k}+w_{x h, k}} \\
& y_{k}=\underbrace{\left[\begin{array}{cc}
C & M
\end{array}\right]}_{C_{x h}} \underbrace{\left[\begin{array}{c}
x_{k} \\
h_{k}
\end{array}\right]}_{x_{x h, k}}+D u_{k}+v_{k}=C_{x h} x_{x h, k}+D u_{k}+v_{k} \\
& z_{k}=\underbrace{\left[\begin{array}{ll}
F & N
\end{array}\right]}_{F_{x h}} \underbrace{\left[\begin{array}{c}
x_{k} \\
h_{k}
\end{array}\right]}_{x_{x h, k}}+G u_{k}=F_{x h} x_{x h, k}+G u_{k}
\end{aligned}
$$

The vector $w_{x h}$ is zero mean white noise associated with the augmented state vector, $\left[x^{T} h^{T}\right]^{T}$, with a covariance of $Q_{x h}$. The vector $w_{x h}$ consists of the original state process noise, $w$, concatenated with the process noise associated with the health parameter vector, $w_{h}$. 


$$
w_{x h, k}=\left[\begin{array}{c}
w_{k} \\
w_{h, k}
\end{array}\right]
$$

The eigenvalues of $A_{x h}$ consist of the original eigenvalues of $A$ plus an additional $\operatorname{dim}(h)$ eigenvalues located at $(1,0)$ on the unit circle due to the augmentation. Thus, the new augmented system given in Equation (3) has at least as many eigenvalues located on the unit circle as there are elements of $h$. Once the $h$ vector is appended to the state vector, it may be directly estimated by a Kalman filter, provided that the realization in Eq. (3) is observable. Using this formulation, the number of health parameters that can be estimated is limited to the number of sensors, the dimension of $y$ (Ref. 4). In an aircraft gas turbine engine there are usually fewer sensors than health parameters, presenting an underdetermined estimation problem. The conventional approach to address this problem is to estimate only a subset of $h$, referred to as tuning parameters. While this enables Kalman filter estimation, it does introduce error within the estimation process due to the effect of those health parameters excluded from estimation. In this paper a departure from the conventional approach of selecting a subset of $h$ to serve as tuning parameters is taken. Instead of selecting the tuning parameter vector to simply consist of a subset of health parameters, this vector is chosen to consist of a linear combination of all health parameters. The tuning parameter will be of dimension equivalent to $y$ in order to facilitate Kalman filter estimation, and will be optimally selected to reduce estimation error within the parameters of interest. The reduced order dynamic state space equations based on the reduced order tuner vector will be introduced in the next subsection.

\section{Reduced Order Dynamic State Space Equations}

Reduced order dynamic state space equations will be constructed based on the model tuning parameter vector, $q$, which is constructed as a linear combination of all health parameters, $h$, and given by

$$
q=V^{*} h
$$

where $q \in \mathbb{R}^{m}, h \in \mathbb{R}^{p}, m<p$, and $V^{*}$ is an $m \times p$ transformation matrix of rank $m$, applied to construct the tuning parameter vector. An approximation of the health parameter vector, $\hat{h}$, can be obtained as

$$
\hat{h}=V^{* \dagger} q
$$

where $V^{* \dagger}$ is the pseudoinverse of $V^{*}$. Substituting Equation (6) into Equation (3) yields the following reduced order state space equations which can be used to formulate the Kalman filter

$$
\begin{aligned}
& {\left[\begin{array}{c}
x_{k+1} \\
q_{k+1}
\end{array}\right]=\underbrace{\left[\begin{array}{cc}
A & L V^{* \dagger} \\
0 & I
\end{array}\right]}_{A_{x q}} \underbrace{\left[\begin{array}{c}
x_{k} \\
q_{k}
\end{array}\right]}_{x_{x q, k}}+\underbrace{\left[\begin{array}{c}
B \\
0
\end{array}\right]}_{B_{x q}} u_{k}+\underbrace{\left[\begin{array}{c}
w_{k} \\
w_{q, k}
\end{array}\right]}_{w_{x q, k}}=A_{x q} x_{x q, k}+B_{x q} u_{k}+w_{x q, k}} \\
& y_{k}=\underbrace{\left[\begin{array}{cc}
C & M V^{* \dagger}
\end{array}\right]}_{C_{x q}} \underbrace{\left[\begin{array}{c}
x_{k} \\
q_{k}
\end{array}\right]}_{x_{x q, k}}+D u_{k}+v_{k}=C_{x q} x_{x q, k}+D u_{k}+v_{k} \\
& z_{k}=\underbrace{\left[\begin{array}{cc}
F & N V^{* \dagger}
\end{array}\right]}_{F_{x q}} \underbrace{\left[\begin{array}{c}
x_{k} \\
q_{k}
\end{array}\right]}_{x_{x q, k}}+G u_{k}=F_{x q} x_{x q, k}+G u_{k}
\end{aligned}
$$

The state process noise, $w_{x q}$, and its associated covariance, $Q_{x q}$, for the reduced order system are calculated as 


$$
\begin{aligned}
w_{x q, k} & =\left[\begin{array}{cc}
I & 0 \\
0 & V^{*}
\end{array}\right] w_{x h, k}=\left[\begin{array}{cc}
I & 0 \\
0 & V^{*}
\end{array}\right]\left[\begin{array}{c}
w_{k} \\
w_{h, k}
\end{array}\right] \\
Q_{x q} & =\left[\begin{array}{cc}
I & 0 \\
0 & V^{*}
\end{array}\right] Q_{x h}\left[\begin{array}{cc}
I & 0 \\
0 & V^{*}
\end{array}\right]^{T}
\end{aligned}
$$

\section{B. Steady-State Measurement Process Equations}

The previous subsection presented state space equations for a dynamic system. Under steady-state operating conditions, these equations reduce to a steady-state measurement process. This subsection will present full order and reduced order steady-state measurement process equations that can be applied to formulate the MAP estimator.

\section{Full Order Steady-State Measurement Process Equations}

Under steady-state open-loop operating conditions, dynamic state space equations reduce to a measurement process of the following form where system outputs are a function of an arbitrary health parameter vector, $h$, as

$$
\begin{aligned}
y_{k} & =H h+v_{k} \\
z & =H_{z} h
\end{aligned}
$$

where $H$ and $H_{z}$ are appropriately dimensioned matrices relating the effects of the health parameter vector to the measured outputs, $y$, and the unmeasured auxiliary parameter outputs, $z$, respectively. The $H$ and $H_{z}$ matrices can be derived from the dynamic state space equations given in Equation (2) by taking advantage of the following expected value properties at steady-state open-loop operating conditions

$$
\begin{aligned}
E\left[x_{k+1}\right] & =E\left[x_{k}\right]=x_{S S} \\
E\left[h_{k}\right] & =h \\
E\left[y_{k}\right] & =y_{s s} \\
E\left[z_{k}\right] & =z_{s s} \\
E\left[u_{k}\right] & =0 \\
E\left[w_{k}\right] & =0 \\
E\left[v_{k}\right] & =0
\end{aligned}
$$

where the subscript "ss" denotes steady-state operation. By taking expected values of Equation (2), $x_{s s}, y_{s s}$ and $z_{s s}$ can be written as functions of the health parameter vector $h$

$$
\begin{aligned}
E\left[x_{k+1}\right] & =A \cdot E\left[x_{k}\right]+B \cdot E\left[u_{k}\right]+L \cdot E\left[h_{k}\right]+E\left[w_{k}\right] \\
x_{s s} & =A x_{s s}+L h \\
x_{s s} & =(I-A)^{-1} L h
\end{aligned}
$$




$$
\begin{aligned}
E\left[y_{k}\right] & =C \cdot E\left[x_{k}\right]+D \cdot E\left[u_{k}\right]+M \cdot E\left[h_{k}\right]+E\left[v_{k}\right] \\
y_{s s} & =C x_{s s}+M h \\
y_{s s} & =\underbrace{\left(C(I-A)^{-1} L+M\right)}_{H} h \\
y_{s S} & =H h \\
E\left[z_{k}\right] & =F \cdot E\left[x_{k}\right]+G \cdot E\left[u_{k}\right]+N \cdot E\left[h_{k}\right] \\
z_{s s} & =F x_{s s}+N h \\
z_{s s} & =\underbrace{\left(F(I-A)^{-1} L+N\right)}_{H_{z}} h \\
z_{S S} & =H_{z} h
\end{aligned}
$$

By including measurement noise effects, Equation (12) becomes $y_{k}=H h+v_{k}$ which, together with Equation (13), provide steady-state system equations of the form given in Equation (9).

\section{Reduced Order Steady-State Measurement Process Equations}

Substituting Equation (6) into Equation (9) yields the following reduced order steady-state measurement process equations

$$
\begin{aligned}
y_{k} & =\underbrace{H V^{* \dagger}}_{H_{q}} q+v_{k} \\
y_{k} & =H_{q} q+v_{k} \\
z & =H_{z} V^{* \dagger} q
\end{aligned}
$$

\section{Kalman Filter Formulation}

Next, the Kalman filter estimator formulation based on the reduced order dynamic state space equations will be presented. In this study, steady-state Kalman filtering is applied. In other words, the state estimation error covariance matrix and the Kalman gain matrix are converged and time invariant. Given the reduced order linear state space equations shown in Equation (7), the state estimation error covariance matrix, $P_{\infty}$, is calculated by solving the following Ricatti equation (Ref. 8)

$$
P_{\infty}=A_{x q} P_{\infty} A_{x q}^{T}-A_{x q} P_{\infty} C_{x q}^{T}\left(C_{x q} P_{\infty} C_{x q}^{T}+R\right)^{-1} C_{x q} P_{\infty} A_{x q}^{T}+Q_{x q}
$$

The steady-state Kalman filter gain, $K_{\infty}$, can then be calculated as follows (Ref. 8)

$$
K_{\infty}=P_{\infty} C_{x q}^{T}\left(C_{x q} P_{\infty} C_{x q}^{T}+R\right)^{-1}
$$

and, assuming steady-state, open-loop operation $(u=0)$, the Kalman filter estimator takes the following form

$$
\hat{x}_{x q, k}=A_{x q} \hat{x}_{x q, k-1}+K_{\infty}\left(y_{k}-C_{x q} A_{x q} \hat{x}_{x q, k-1}\right)
$$


The reduced order state vector estimate, $\hat{x}_{x q}$, produced by Equation (17) can be used to produce an estimate of the state variables, health parameters, and the auxiliary parameter vector as follows

$$
\begin{aligned}
{\left[\begin{array}{c}
\hat{x}_{k} \\
\hat{h}_{k}
\end{array}\right] } & =\left[\begin{array}{cc}
I & 0 \\
0 & V^{* \dagger}
\end{array}\right] \hat{x}_{x q, k} \\
\hat{z}_{k} & =\left[\begin{array}{ll}
F & N V^{* \dagger}
\end{array}\right] \hat{x}_{x q, k}
\end{aligned}
$$

\section{Maximum a Posteriori Estimator Formulation}

The maximum a posteriori (MAP) estimator can be formulated based on the steady-state measurement process equations given in Equations (12) and (13). The MAP estimator follows the closed form expression (Ref. 9)

$$
\hat{h}_{k}=\left(P_{h}^{-1}+H^{T} R^{-1} H\right)^{-1} H^{T} R^{-1} y_{k}
$$

where the matrix $P_{h}$, defined as $E\left[h h^{T}\right]$, reflects a priori or historical knowledge of the covariance in the health parameters across all engines. The MAP estimate of the health parameter vector can be used to produce an estimate of the auxiliary parameter vector by premultiplying the right side of Equation (19) by $H_{z}$

$$
\begin{aligned}
& \hat{z}_{k}=H_{z} \hat{h}_{k} \\
& \hat{z}_{k}=H_{z}\left(P_{h}^{-1}+H^{T} R^{-1} H\right)^{-1} H^{T} R^{-1} y_{k}
\end{aligned}
$$

A MAP estimate of the reduced order tuner vector, $q$, can also be formulated based on the reduced order steady-state measurement process model given in Equation (14). This reduced order estimator is given as

$$
\hat{q}_{k}=\left(P_{q}^{-1}+H_{q}^{T} R^{-1} H_{q}\right)^{-1} H_{q}^{T} R^{-1} y_{k}
$$

where $P_{q}$, defined as $E\left[q q^{T}\right]$, is the covariance in the tuner vector. It is calculated as

$$
P_{q}=V^{*} P_{h} V^{* T}
$$

Once the estimate of $\hat{q}_{k}$ is obtained from Equation (21), estimates of the health parameter vector and the auxiliary parameter vector can be obtained as

$$
\begin{aligned}
\hat{h}_{k} & =V^{* \dagger} \hat{q}_{k} \\
\hat{h}_{k} & =V^{* \dagger} \underbrace{\left(P_{q}^{-1}+H_{q}^{T} R^{-1} H_{q}\right)^{-1} H_{q}^{T} R^{-1} y_{k}}_{\hat{q}_{k}} \\
\hat{z}_{k} & =H_{z} V^{* \dagger} \hat{q}_{k} \\
& =H_{z} V^{* \dagger} \underbrace{\left(P_{q}^{-1}+H_{q}^{T} R^{-1} H_{q}\right)^{-1} H_{q}^{T} R^{-1} y_{k}}_{\hat{q}_{k}}
\end{aligned}
$$




\section{E. Analytical Derivation of MAP Estimation Error}

The estimation errors in $\hat{h}_{k}$ and $\hat{z}_{k}$ are defined as the difference between these estimates and their corresponding actual values

$$
\begin{aligned}
& \tilde{h}_{k}=\hat{h}_{k}-h_{k} \\
& \tilde{z}_{k}=\hat{z}_{k}-z_{k}
\end{aligned}
$$

The estimation errors can be considered to consist of two components: an estimation error bias, and an estimation variance. Due to the underdetermined nature of the problem, the MAP estimator will be a biased estimator (i.e. the expected values of $\tilde{h}_{k}$ and $\tilde{z}_{k}$ will be non-zero). The estimation error bias vector is equivalent to the mean estimation error vector defined as

$$
\begin{aligned}
& \overline{\tilde{h}}_{k}=E\left[\tilde{h}_{k}\right]=E\left[\hat{h}_{k}-h_{k}\right] \\
& \overline{\tilde{z}}_{k}=E\left[\tilde{z}_{k}\right]=E\left[\hat{z}_{k}-z_{k}\right]
\end{aligned}
$$

The variance of the estimates can be found by constructing their respective estimation covariance matrices

$$
\begin{aligned}
& P_{\hat{h}, k}=E\left[\left(\hat{h}_{k}-E\left[\hat{h}_{k}\right]\right)\left(\hat{h}_{k}-E\left[\hat{h}_{k}\right]\right)^{T}\right] \\
& P_{\hat{z}, k}=E\left[\left(\hat{z}_{k}-E\left[\hat{z}_{k}\right]\right)\left(\hat{z}_{k}-E\left[\hat{z}_{k}\right]\right)^{T}\right]
\end{aligned}
$$

Diagonal elements of the covariance matrices will reflect the variance in individual parameter estimates, while off diagonal elements reflect the covariance between parameter estimates.

The overall sum of squared estimation errors (SSEE) can be obtained by combining the estimation error bias and estimation variance information as

$$
\begin{aligned}
& \operatorname{SSEE}\left(\hat{h}_{k}\right)=\overline{\tilde{h}}_{k}^{T} \overline{\tilde{h}}_{k}+\operatorname{tr}\left\{P_{\hat{h}_{, k}}\right\} \\
& \operatorname{SSEE}\left(\hat{z}_{k}\right)=\overline{\tilde{z}}_{k}^{T} \overline{\tilde{z}}_{k}+\operatorname{tr}\left\{P_{\hat{z}, k}\right\}
\end{aligned}
$$

where $\operatorname{tr}\{\cdot\}$ represents the trace (sum of the diagonal elements) of the matrix. In this paper, minimization of the SSEE will be applied as the metric used to facilitate optimal tuner parameter selection. First, theoretical values for each component of the MAP SSEE, estimation error bias and estimation variance, will be derived assuming reduced order tuner estimation under steady-state, open-loop $(u=0)$ operating conditions. These derivations are provided in the following subsections.

\section{Analytical Derivation of MAP Estimation Error Bias}

The estimation error biases, $\overline{\tilde{h}}_{k}$ and $\overline{\tilde{z}}_{k}$, when applying reduced order tuner estimation, can be analytically derived for an arbitrary health parameter vector, $h$, at steady-state operating conditions. First, by taking expected values of both sides of Equation (23) the expected steady-state values of $\hat{h}_{k}$ and $\hat{z}_{k}$ can be written as a function of $h$ 


$$
\begin{aligned}
E\left[\hat{h}_{k}\right] & =V^{* \dagger}\left(P_{q}^{-1}+H_{q}^{T} R^{-1} H_{q}\right)^{-1} H_{q}^{T} R^{-1} \cdot E\left[y_{k}\right] \\
\overline{\hat{h}}_{s s} & =V^{* \dagger}\left(P_{q}^{-1}+H_{q}^{T} R^{-1} H_{q}\right)^{-1} H_{q}^{T} R^{-1} y_{s s} \\
\overline{\hat{h}}_{s s} & =V^{* \dagger}\left(P_{q}^{-1}+H_{q}^{T} R^{-1} H_{q}\right)^{-1} H_{q}^{T} R^{-1} H h \\
E\left[\hat{z}_{k}\right] & =H_{z} V^{* \dagger}\left(P_{q}^{-1}+H_{q}^{T} R^{-1} H_{q}\right)^{-1} H_{q}^{T} R^{-1} \cdot E\left[y_{k}\right] \\
\overline{\hat{z}}_{s s} & =H_{z} V^{* \dagger}\left(P_{q}^{-1}+H_{q}^{T} R^{-1} H_{q}\right)^{-1} H_{q}^{T} R^{-1} y_{s s} \\
\overline{\hat{z}}_{s s} & =H_{z} V^{* \dagger}\left(P_{q}^{-1}+H_{q}^{T} R^{-1} H_{q}\right)^{-1} H_{q}^{T} R^{-1} H h
\end{aligned}
$$

The steady-state estimation error biases, $\overline{\tilde{h}}_{S S}$ and $\overline{\tilde{z}}_{S S}$, can then be written as

$$
\begin{aligned}
\overline{\tilde{h}}_{s s} & =E\left[\hat{h}_{k}-h\right] \\
& =\overline{\hat{h}}_{s S}-h \\
& =\underbrace{V^{* \dagger}\left(P_{q}^{-1}+H_{q}^{T} R^{-1} H_{q}\right)^{-1} H_{q}^{T} R^{-1} H h}_{\hat{\hat{h}}_{s s}}-h \\
& =\underbrace{\left(V^{* \dagger}\left(P_{q}^{-1}+H_{q}^{T} R^{-1} H_{q}\right)^{-1} H_{q}^{T} R^{-1} H-I\right)}_{G_{h}} h \\
& =\overline{\tilde{z}}_{S S}=\underbrace{E\left[\hat{z}_{k}-z\right]}_{\hat{\hat{z}}_{s S}} \\
& =\overline{\hat{z}}_{S S}-z \\
& =\underbrace{H_{z} V^{* \dagger}\left(P_{q}^{-1}+H_{q}^{T} R^{-1} H_{q}\right)^{-1} H_{q}^{T} R^{-1} H h}_{G_{z}}-\underbrace{H_{z} h}_{z} \\
& =\underbrace{\left(H_{z} V^{* \dagger}\left(P_{q}^{-1}+H_{q}^{T} R^{-1} H_{q}\right)^{-1} H_{q}^{T} R^{-1} H\right.}-H_{z}^{\left.H_{z}\right)} h \\
& =G_{z} h
\end{aligned}
$$

The steady-state estimation error bias equations given in Equation (29) are functions of an arbitrary health parameter vector, $h$. As such they are representative of the parameter estimation error biases in a single engine, at a given point in its lifetime of use where its deterioration is represented by the health parameter vector $h$. The average sum of squared estimation error biases across a fleet of engines can be calculated as 


$$
\begin{aligned}
\overline{\tilde{h}}_{\text {fleet }}^{2} \equiv E\left[\overline{\tilde{h}}_{S S}^{T} \overline{\tilde{h}}_{s s}\right] & =E\left[\operatorname{tr}\left\{\overline{\tilde{h}}_{s s} \overline{\tilde{h}}_{S S}^{T}\right\}\right] \\
& =E\left[\operatorname{tr}\left\{G_{h} h h^{T} G_{h}^{T}\right\}\right] \\
& =\operatorname{tr}\{G_{h} \cdot \underbrace{E\left[h h^{T}\right]}_{P_{h}} G_{h}^{T}\} \\
& =\operatorname{tr}\left\{G_{h} P_{h} G_{h}^{T}\right\} \\
\overline{\tilde{z}}_{\text {fleet }}^{2} \equiv E\left[\overline{\tilde{z}}_{S S}^{T} \overline{\tilde{z}}_{S S}\right] & =E\left[\operatorname{tr}\left\{\overline{\tilde{z}}_{s S} \overline{\tilde{z}}_{S S}^{T}\right\}\right] \\
& =E\left[\operatorname{tr}\left\{G_{z} h h^{T} G_{z}^{T}\right\}\right] \\
& =\operatorname{tr}\{G_{z} \cdot \underbrace{E\left[G_{z}^{T}\right.}_{P_{h}\left[h h^{T}\right]}\} \\
& =\operatorname{tr}\left\{G_{z} P_{h} G_{z}^{T}\right\}
\end{aligned}
$$

\section{Analytical Derivation of MAP Estimation Variance}

Next, the variance of the health parameter and auxiliary parameter estimates is derived. First consider the health parameter estimate covariance matrices, $P_{\hat{h}, k}$, defined as

$$
P_{\hat{h}, k}=E[\underbrace{\left(\hat{h}_{k}-E\left[\hat{h}_{k}\right]\right)}_{\varepsilon_{k}} \underbrace{\left(\hat{h}_{k}-E\left[\hat{h}_{k}\right]\right)^{T}}_{\varepsilon_{k}^{T}}]
$$

where the vector $\varepsilon_{k}$ is defined as the residual between $\hat{h}_{k}$ at time $k$ and its expected value. Since $E\left[\hat{h}_{k}\right]=\overline{\hat{h}}_{s S}, \varepsilon_{k}$ can be obtained by subtracting Equation (28) from Equation (23)

$$
\begin{aligned}
\varepsilon_{k} & =\hat{h}_{k}-E\left[\hat{h}_{k}\right] \\
& =\hat{h}_{k}-\overline{\hat{h}}_{s s} \\
& =\underbrace{V^{*} \dagger\left(P_{q}^{-1}+H_{q}^{T} R^{-1} H_{q}\right)^{-1} H_{q}^{T} R^{-1} y_{k}}_{\hat{h}_{k}}-\underbrace{V^{* \dagger}\left(P_{q}^{-1}+H_{q}^{T} R^{-1} H_{q}\right)^{-1} H_{q}^{T} R^{-1} y_{s s}}_{\hat{h}_{s s}} \\
& =V^{* \dagger}\left(P_{q}^{-1}+H_{q}^{T} R^{-1} H_{q}\right)^{-1} H_{q}^{T} R^{-1}\left(y_{k}-y_{s s}\right)
\end{aligned}
$$

Making the substitution $v_{k}=y_{k}-y_{s s}$ yields 


$$
\varepsilon_{k}=V^{* \dagger}\left(P_{q}^{-1}+H_{q}^{T} R^{-1} H_{q}\right)^{-1} H_{q}^{T} R^{-1} v_{k}
$$

The estimation covariance matrix $P_{\hat{h}, k}$ is then calculated as

$$
\begin{aligned}
P_{\hat{h}, k} & =E\left[\varepsilon_{k} \varepsilon_{k}^{T}\right] \\
& =\left(V^{* \dagger}\left(P_{q}^{-1}+H_{q}^{T} R^{-1} H_{q}\right)^{-1} H_{q}^{T} R^{-1}\right) \cdot \underbrace{E\left[v_{k} v_{k}^{T}\right]}_{R} \cdot\left(V^{* \dagger}\left(P_{q}^{-1}+H_{q}^{T} R^{-1} H_{q}\right)^{-1} H_{q}^{T} R^{-1}\right)^{T} \\
& =\left(V^{* \dagger}\left(P_{q}^{-1}+H_{q}^{T} R^{-1} H_{q}\right)^{-1} H_{q}^{T} R^{-1}\right) R\left(V^{* \dagger}\left(P_{q}^{-1}+H_{q}^{T} R^{-1} H_{q}\right)^{-1} H_{q}^{T} R^{-1}\right)^{T}
\end{aligned}
$$

Applying a similar derivation, the auxiliary parameter estimate covariance matrix, $P_{\hat{z}, k}$, can be obtained as

$$
P_{\hat{z}, k}=\left(H_{z} V^{* \dagger}\left(P_{q}^{-1}+H_{q}^{T} R^{-1} H_{q}\right)^{-1} H_{q}^{T} R^{-1}\right) R\left(H_{z} V^{* \dagger}\left(P_{q}^{-1}+H_{q}^{T} R^{-1} H_{q}\right)^{-1} H_{q}^{T} R^{-1}\right)^{T}
$$

The variance in the estimates $\hat{h}_{k}$ and $\hat{z}_{k}$ can be obtained from the diagonals of the covariance matrices produced by Equations (35) and (36) respectively.

\section{Analytical Derivation of MAP Sum of Squared Estimation Errors}

Once Equations (30), (31), (35), and (36) are obtained, they may be used to analytically calculate the mean sum of squared estimation errors over all engines by combining the respective estimation error bias and estimation variance information as previously shown in Equation (27). The health parameter sum of squared estimation errors, $\operatorname{SSEE}\left(\hat{h}_{\text {fleet }}\right)$, and the auxiliary parameter vector sum of squared estimation errors, $\operatorname{SSEE}\left(\hat{z}_{\text {fleet }}\right)$, become

$$
\begin{aligned}
\operatorname{SSEE}\left(\hat{h}_{\text {fleet }}\right) & =\overline{\tilde{h}}_{\text {fleet }}^{2}+\operatorname{tr}\left\{P_{\hat{h}, k}\right\} \\
& =\operatorname{tr}\left\{G_{h} P_{h} G_{h}^{T}+P_{\hat{h}, k}\right\} \\
\operatorname{SSEE}\left(\hat{z}_{\text {fleet }}\right) & =\overline{\tilde{z}}_{\text {fleet }}^{2}+\operatorname{tr}\left\{P_{\hat{z}, k}\right\} \\
& =\operatorname{tr}\left\{G_{z} P_{h} G_{z}^{T}+P_{\hat{z}, k}\right\}
\end{aligned}
$$

From Equations (29), (35), and (36) it can be observed that both bias and variance are affected by the selection of the transformation matrix, $V^{*}$. The sum of squared estimation error terms derived in this section (Eq. (37)) give rise to an optimization problem: selecting $V^{*}$ to minimize the squared estimation error in the MAP-produced parameter estimates. These parameter estimates could include health parameter estimates, auxiliary parameter estimates, or a combination of parameters. Although there is no known closed form solution for optimally selecting the $V^{*}$ matrix to satisfy the objective of minimizing estimation errors, a multi-parameter iterative search method has been developed to perform this task, and will be described in the next section.

\section{F. Optimal Transformation Matrix Selection}

Prior to initiating the search for an optimal $V^{*}$, specific system design information must be defined or obtained. This includes: 
- $\quad$ Specifying the auxiliary parameters, $z$, to be estimated

- Generating system state space equations (Eq. (2)) at a fleet average (50 percent deteriorated) engine trim point. The result is expected values of zero for the health parameters and measured outputs, which is consistent with the theoretical derivations provided in this paper.

- Defining measurement noise covariance matrix, $R$

- Defining fleet average health parameter covariance, $P_{h}$

After the necessary system information has been obtained, the search for an optimal transformation matrix to minimize the MAP sum of squared estimation errors can commence. The process is performed using the lsqnonlin function of the Matlab (The MathWorks, Inc.) Optimization Toolbox. This function applies an iterative search to find the least squares solution of a user specified multivariable optimization problem. A further description of each step of this optimal iterative search is given below.

1. Upon startup, an initial random guess of the transformation matrix, $V^{*}$, is generated.

2. The pseudoinverse of the transformation matrix, $V^{* \dagger}$, is calculated for implementation within the estimator, and for estimator error calculation purposes. $V^{* \dagger}$ can be calculated by the Matlab pinv function.

3. Construct the reduced order steady-state measurement process model (Eq. (14)).

4. Formulate the reduced order MAP estimator (Eq. (23)).

5. Calculate sum of squared estimation errors, SSEE (Eq. (37)).

6. On each iteration, the change in SSEE relative to the previous iteration is assessed to determine if convergence within a user specified tolerance has been achieved.

a. If converged, skip step 7 and proceed to step 8 .

b. If not converged, proceed to step 7 to update $V^{*}$.

7. $V^{*}$ is updated via the Matlab lsqnonlin function, and the process returns to step 2.

8. Upon convergence, the optimization routine returns the optimal value of $V^{*}$, and ends.

Experience has shown that the transformation matrix returned by the optimization routine is not unique - different matrices can be found that provide equivalent estimation accuracy. Experience has also shown that the optimization routine will usually return a $V^{*}$ matrix that satisfies, or nearly satisfies (i.e. within 2 percent), the global minimum of the objective function. However, in order to guard against potential convergence to a local minimum, it is prudent to run the optimization routine multiple times, each time starting with a different initial guess for $V^{*}$. This is only to assure the designer that the global minimum is achieved, not to produce a consistent $V^{*}$. It should be emphasized that the optimal search for $V^{*}$ is only conducted offline during the estimator design process. This calculation is not conducted as part of the online estimator implementation, and thus places no additional computational burden upon it.

\section{Turbofan Engine Example}

A linearized cruise operating point extracted from the NASA Commercial Modular Aero-Propulsion System Simulation (C-MAPSS (Ref. 10)) high bypass turbofan engine model is used to evaluate the tuner selection methodology. The linear model has two state variables, ten health parameters, and three control inputs, all shown in Table 1. The model's seven sensed outputs, and corresponding sensor noise standard deviation, are shown in Table 2. The auxiliary output parameters of interest to be estimated are shown in Table 3. 
TABLE 1.-STATE VARIABLES, HEALTH PARAMETERS, AND ACTUATORS

\begin{tabular}{|c|c|c|}
\hline State variables & Health parameters & Actuators \\
\hline $\begin{array}{l}\mathrm{Nf} \text { - fan speed } \\
\mathrm{Nc} \text { - core speed }\end{array}$ & $\begin{array}{l}\eta_{\text {FAN }}-\text { Fan efficiency } \\
\gamma_{\text {FAN }}-\text { Fan flow capacity } \\
\eta_{\text {LPC }}-\text { LPC efficiency } \\
\gamma_{\text {LPC }}-\text { LPC flow capacity } \\
\eta_{\text {HPC }}-\text { HPC efficiency } \\
\gamma_{\mathrm{HPC}}-\text { HPC flow capacity } \\
\eta_{\text {HPT }}-\text { HPT efficiency } \\
\gamma_{\text {HPT }}-\text { HPT flow capacity } \\
\eta_{\text {LPT }}-\text { LPT efficiency } \\
\gamma_{\text {LPT }} \text {-LPT flow capacity }\end{array}$ & $\begin{array}{l}\text { Wf-fuel flow } \\
\text { VSV-variable stator vane } \\
\text { VBV_-variable bleed valve }\end{array}$ \\
\hline
\end{tabular}

TABLE 2.-SENSED OUTPUTS AND STANDARD DEVIATION

IN PERCENT OF OPERATING POINT TRIM VALUES

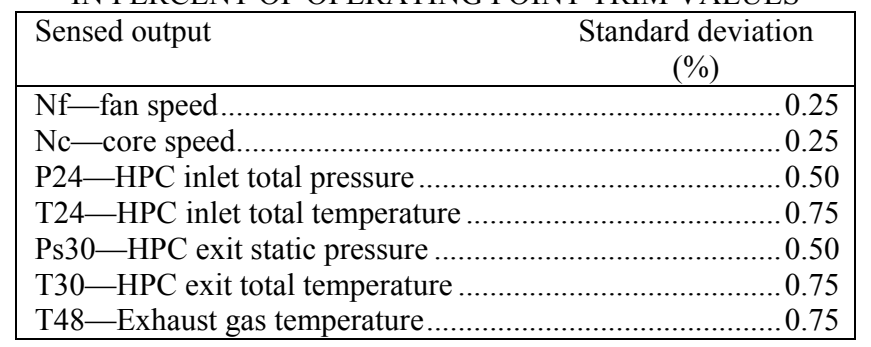

TABLE 3.-ESTIMATED AUXILIARY PARAMETERS

\begin{tabular}{|c|}
\hline Auxiliary parameter \\
\hline ............................. Combustor exit temperature \\
\hline ...............PT exit temperature \\
\hline 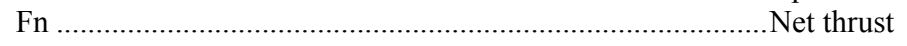 \\
\hline 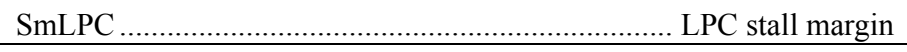 \\
\hline
\end{tabular}

Next, the estimation accuracy achievable by estimating the systematically selected reduced order tuner vectors will be assessed. This assessment will be conducted for both MAP estimation and Kalman filter estimation.

\section{A. MAP Estimation Accuracy Comparison}

A linear steady-state measurement process model-based on Equations (12) and (13) is used as the truth model for this application example. Deviations in all ten health parameter inputs are assumed to be uncorrelated and normally distributed about their trim conditions with a standard deviation of 0.02 (2 percent). Since a parameter's variance is equal to its standard deviation squared, the health parameter covariance matrix, $P_{h}$, is defined as a diagonal matrix with all diagonal elements equal to 0.0004 . The iterative search for a transformation matrix and associated reduced order tuner vector optimal for MAP estimation is run multiple times, each time starting with a different initial random guess of $V^{*}$. This is done so that the estimation consistency enabled by the $q$ vectors returned by the iterative search routine can be assessed. First, four different $q$ vectors are optimally selected to minimize the MAP estimated health parameter mean squared error. These four vectors are denoted as $a, b, c$ and $d$. Then the process is repeated and four additional $q$ vectors are selected, this time optimized for minimizing the MAP estimated auxiliary parameter mean squared error. These four vectors are denoted as e, $\mathrm{f}, \mathrm{g}$, and $\mathrm{h}$.

The health parameter estimation error results are summarized in Table 4 for MAP estimation of all 10 health parameters directly through Equation (19), or indirectly through reduced order tuner vectors as given in Equation (23). This table shows the theoretically predicted and experimentally obtained percent squared estimation errors for each case. The experimental results are obtained through a Monte Carlo simulation analysis with random health parameter deviations normally distributed with covariance 
equivalent to $P_{h}$. A total of 100,000 test cases are produced and evaluated using each approach. It can be seen that the theoretically predicted and the experimentally obtained percent squared estimation errors exhibit good agreement. Based on Table 4 it can be seen that the four $q$ vectors optimal for health parameter estimation, (vectors a, b, c and d), are fairly consistent in terms of the MAP estimation accuracy that they provide. Estimating all 10 health parameters directly results in a theoretical sum of squared estimation errors (SSEE) of 21.48, while estimating the reduced order (seven element) $q$ vectors results in theoretical SSEE's ranging from 21.73 to 21.95 , approximately 1.1 to 2.2 percent more error than estimating all 10 health parameters. Also shown in Table 4 is the MAP health parameter estimation accuracy provided by the four $q$ vectors optimally selected for auxiliary parameter estimation, vectors e, f, $\mathrm{g}$ and $\mathrm{h}$. The estimation errors based on these tuners are larger and exhibit more variance. Their theoretical SSEE's range from 23.90 to 26.13 , or 11 to 22 percent larger than the error in estimating all 10 health parameters directly. However, this result is not surprising considering that these tuners are optimized for reducing auxiliary parameter estimation error, not health parameter estimation error.

TABLE 4.-MAP ESTIMATOR HEALTH PARAMETER PERCENT SQUARED ESTIMATION ERROR

\begin{tabular}{|c|c|c|c|c|c|c|c|c|c|c|c|c|c|}
\hline $\begin{array}{l}\text { Estimated } \\
\text { parameters }\end{array}$ & $\begin{array}{l}\text { Tuner } \\
\text { vector }\end{array}$ & $\begin{array}{c}\text { Theory/ } \\
\text { experiment }\end{array}$ & $\eta_{\text {FAN }}$ & $\gamma_{\mathrm{FAN}}$ & $\eta_{\mathrm{LPC}}$ & $\gamma_{\mathrm{LPC}}$ & $\eta_{\text {HPC }}$ & $\gamma_{\mathrm{HPC}}$ & $\eta_{\mathrm{HPT}}$ & $\gamma_{\mathrm{HPT}}$ & $\eta_{\mathrm{LPT}}$ & $\gamma_{\mathrm{LPT}}$ & $\operatorname{SSEE}(\hat{h})$ \\
\hline \multirow{2}{*}{$\begin{array}{l}10 \text { health } \\
\text { parameters }\end{array}$} & \multirow{2}{*}{ N/A } & Theory & 2.63 & 2.02 & 3.69 & 2.54 & 1.16 & 1.67 & 1.36 & 0.52 & 2.64 & 3.26 & 21.48 \\
\hline & & Experiment & 2.62 & 2.03 & 3.69 & 2.55 & 1.15 & 1.66 & 1.36 & 0.52 & 2.64 & 3.27 & 21.50 \\
\hline \multirow{8}{*}{$\begin{array}{l}7 \text { element } \\
\text { reduced } \\
\text { order tuner } \\
\text { vector } \\
\text { selected to } \\
\text { minimize } h \\
\text { estimation } \\
\text { error }\end{array}$} & \multirow{2}{*}{$\mathrm{a}$} & Theory & 2.66 & 2.04 & 3.76 & 2.56 & 1.16 & 1.68 & 1.37 & 0.52 & 2.69 & 3.31 & 21.73 \\
\hline & & Experiment & 2.66 & 2.05 & 3.75 & 2.57 & 1.16 & 1.67 & 1.37 & 0.52 & 2.68 & 3.32 & 21.73 \\
\hline & \multirow[b]{2}{*}{$\mathrm{b}$} & Theory & 2.66 & 2.04 & 3.89 & 2.54 & 1.16 & 1.75 & 1.36 & 0.52 & 2.74 & 3.27 & 21.95 \\
\hline & & Experiment & 2.66 & 2.05 & 3.90 & 2.56 & 1.16 & 1.74 & 1.36 & 0.52 & 2.73 & 3.28 & 21.96 \\
\hline & \multirow{2}{*}{ c } & Theory & 2.79 & 2.04 & 3.71 & 2.56 & 1.16 & 1.70 & 1.37 & 0.52 & 2.72 & 3.32 & 21.89 \\
\hline & & Experiment & 2.78 & 2.05 & 3.71 & 2.57 & 1.16 & 1.69 & 1.38 & 0.52 & 2.71 & 3.33 & 21.90 \\
\hline & \multirow{2}{*}{ d } & Theory & 2.67 & 2.03 & 3.79 & 2.57 & 1.16 & 1.71 & 1.39 & 0.52 & 2.65 & 3.36 & 21.84 \\
\hline & & Experiment & 2.66 & 2.04 & 3.79 & 2.58 & 1.16 & 1.70 & 1.39 & 0.52 & 2.65 & 3.37 & 21.86 \\
\hline \multirow{8}{*}{$\begin{array}{l}7 \text { element } \\
\text { reduced } \\
\text { order tuner } \\
\text { vector } \\
\text { selected to } \\
\text { minimize } z \\
\text { estimation } \\
\text { error }\end{array}$} & \multirow[b]{2}{*}{$\mathrm{e}$} & Theory & 2.66 & 2.03 & 4.65 & 2.69 & 1.39 & 2.23 & 1.50 & 0.57 & 2.68 & 3.50 & 23.90 \\
\hline & & Experiment & 2.65 & 2.04 & 4.65 & 2.70 & 1.39 & 2.22 & 1.50 & 0.57 & 2.69 & 3.52 & 23.94 \\
\hline & \multirow{2}{*}{$\mathrm{f}$} & Theory & 3.06 & 2.19 & 4.45 & 2.67 & 1.72 & 2.65 & 1.86 & 0.58 & 2.86 & 4.09 & 26.13 \\
\hline & & Experiment & 3.05 & 2.21 & 4.44 & 2.68 & 1.72 & 2.64 & 1.86 & 0.59 & 2.86 & 4.12 & 26.19 \\
\hline & \multirow[b]{2}{*}{8} & Theory & 3.87 & 2.65 & 4.12 & 2.56 & 1.34 & 1.72 & 1.40 & 0.53 & 2.65 & 3.62 & 24.48 \\
\hline & & Experiment & 3.89 & 2.67 & 4.11 & 2.58 & 1.33 & 1.71 & 1.40 & 0.54 & 2.65 & 3.64 & 24.52 \\
\hline & \multirow{2}{*}{$\mathrm{h}$} & Theory & 2.69 & 2.09 & 4.78 & 2.65 & 1.20 & 1.76 & 1.75 & 0.54 & 2.80 & 3.76 & 24.01 \\
\hline & & Experiment & 2.68 & 2.11 & 4.80 & 2.66 & 1.20 & 1.75 & 1.75 & 0.54 & 2.80 & 3.77 & 24.05 \\
\hline
\end{tabular}

Table 5 shows a comparison of theoretical and experimental auxiliary parameter estimation error results obtained via MAP estimation. The experimental results are based on the same 100,000 Monte Carlo simulation runs used to generate health parameter estimation results previously shown in Table 4. Those results based on the estimation of all 10 health parameters are obtained through Equation (20), while the results based on reduced order tuner estimation are obtained through Equation (23). Once again the theoretical and experimental estimation accuracy is found to exhibit good agreement. Auxiliary parameter estimates based on a MAP estimate of all 10 health parameters have a theoretical SSEE of 363.86. Estimating the four $q$ vectors optimized for health parameter estimation (vectors a, b, c, and d) have theoretical SSEE's ranging from 366.13 to 371.16 , a 0.6 to 2.0 percent increase in error compared to that obtained by estimating all 10 health parameters. This is comparable to the health parameter estimation accuracy found in Table 4 . The four $q$ vectors optimally selected for auxiliary parameter estimation (vectors e, $\mathrm{f}, \mathrm{g}$ and $\mathrm{h}$ ) result in theoretical SSEE's ranging from 363.89 to 364.12 , only 0.01 to 0.07 percent more error than that found when estimating all 10 health parameters. These results reveal two significant findings. First, the $q$ vectors optimally selected for auxiliary parameter estimation only come close to matching, not exceeding, the auxiliary parameter estimation accuracy obtained by estimating all 10 health parameters. Second, the $q$ vectors optimally selected for health parameter estimation (vectors a, b, c, and d) appear to perform reasonably well in minimizing auxiliary parameter estimation errors. This last result is particularly significant because it implies that the application of a 
single estimator which satisfies the dual objective of estimating both health parameters and auxiliary parameters might be feasible. However, additional study beyond this single example is required to determine if this consistently holds true, particularly when considering estimation of different or expanded auxiliary parameter vectors.

\begin{tabular}{|c|c|c|c|c|c|c|c|}
\hline $\begin{array}{c}\text { Estimated } \\
\text { parameters }\end{array}$ & $\begin{array}{l}\text { Tuner } \\
\text { vector }\end{array}$ & $\begin{array}{c}\text { Theory / } \\
\text { experiment }\end{array}$ & $\begin{array}{l}\mathrm{T} 40 \\
\left({ }^{\circ} \mathrm{R}\right)\end{array}$ & $\begin{array}{l}\mathrm{T} 50 \\
\left({ }^{\circ} \mathrm{R}\right)\end{array}$ & $\begin{array}{l}\text { Fn } \\
(\%)\end{array}$ & $\begin{array}{c}\text { SmLPC } \\
(\%)\end{array}$ & $\operatorname{SSEE}(\hat{z})$ \\
\hline \multirow{2}{*}{$\begin{array}{l}\text { All } 10 \text { health } \\
\text { parameters }\end{array}$} & \multirow{2}{*}{ N/A } & Theory & 193.49 & 167.15 & 0.96 & 2.26 & 363.86 \\
\hline & & Experiment & 195.61 & 167.63 & 0.96 & 2.27 & 366.47 \\
\hline \multirow{8}{*}{$\begin{array}{l}7 \text { element } \\
\text { reduced order } \\
\text { tuner vector } \\
\text { selected to } \\
\text { minimize h error }\end{array}$} & \multirow{2}{*}{$\mathrm{a}$} & Theory & 193.55 & 170.10 & 0.97 & 2.27 & 366.90 \\
\hline & & Experiment & 195.63 & 170.18 & 0.97 & 2.28 & 369.06 \\
\hline & \multirow{2}{*}{$b$} & Theory & 194.73 & 173.19 & 0.97 & 2.27 & 371.16 \\
\hline & & Experiment & 196.56 & 173.09 & 0.97 & 2.27 & 372.89 \\
\hline & \multirow{2}{*}{$\mathrm{c}$} & Theory & 193.52 & 172.54 & 0.97 & 2.27 & 369.30 \\
\hline & & Experiment & 195.59 & 172.40 & 0.97 & 2.28 & 371.24 \\
\hline & \multirow{2}{*}{ d } & Theory & 194.04 & 168.85 & 0.96 & 2.27 & 366.13 \\
\hline & & Experiment & 195.94 & 168.97 & 0.97 & 2.28 & 368.16 \\
\hline \multirow{8}{*}{$\begin{array}{l}7 \text { element } \\
\text { reduced order } \\
\text { tuner vector } \\
\text { selected to } \\
\text { minimize } \mathrm{z} \text { error }\end{array}$} & \multirow{2}{*}{ e } & Theory & 193.49 & 167.16 & 0.96 & 2.28 & 363.89 \\
\hline & & Experiment & 195.61 & 167.66 & 0.97 & 2.29 & 366.52 \\
\hline & \multirow[b]{2}{*}{1} & Theory & 193.49 & 167.15 & 0.97 & 2.52 & 364.12 \\
\hline & & Experiment & 195.61 & 167.63 & 0.97 & 2.52 & 366.74 \\
\hline & \multirow[b]{2}{*}{ g } & Theory & 193.49 & 167.15 & 1.10 & 2.27 & 364.01 \\
\hline & & Experiment & 195.61 & 167.66 & 1.10 & 2.28 & 366.65 \\
\hline & & Theory & 193.49 & 167.15 & 0.97 & 2.31 & 363.93 \\
\hline & & Experiment & 195.60 & 167.65 & 0.98 & 2.32 & 366.55 \\
\hline
\end{tabular}

\section{B. Kalman Filter Estimation Accuracy Comparison}

The results presented in the previous subsection demonstrate that an optimally selected reduced order tuner vector can be found that approaches the full order health parameter vector in terms of MAP estimator accuracy. However, readers are reminded that the actual objective for pursing this effort is to produce a reduced order vector that minimizes Kalman filter estimation errors when presented the underdetermined estimation problem of having fewer sensors than unknown health parameters. Towards that objective, the estimation accuracy of a Kalman filter using the previously selected reduced order tuner vectors is compared to the estimation accuracy obtained by applying the conventional approach of selecting a subset of health parameters to serve as Kalman filter tuners. These experimental estimation results are obtained through a Monte Carlo simulation analysis where the health parameter vectors are normally distributed in accordance with the covariance matrix, $P_{h}$. These health parameter test cases are provided as inputs to the C-MAPSS linear discrete state space model given in Equation (2), with an update rate of $15 \mathrm{~ms}$. In order to provide the recursive Kalman filter estimator ample time to reach convergence each test case is $30 \mathrm{~s}$ in duration. The estimation errors are determined by calculating the mean squared error between estimated and actual values during the last $10 \mathrm{~s}$ of each $30 \mathrm{~s}$ test case. The error calculation is based on only the last $10 \mathrm{~s}$ so that engine model outputs and Kalman estimator outputs have reached a quasi-steady-state operating condition prior to calculating the error. A total of $37530 \mathrm{~s}$ test cases are evaluated.

Table 6 shows a comparison of the experimental squared health parameter estimation errors obtained using a Kalman filter. This includes results obtained from applying the conventional approach of estimating a subset of health parameters (the seven health parameters denoted with an "*" in the table, which are identical to the subset of health parameters selected as tuners in Ref. 5), and from estimating the eight reduced order tuner vectors (vectors a through $h$ ) previously selected for MAP estimation. This table shows that the experimental health parameter SSEE obtained by estimating the subset of seven health parameters is 200.9. In this case, the estimated health parameters that comprise the subset are treated as tuning parameters that are adjusted by the Kalman filter to enable it to produce estimated 
outputs that match sensed engine outputs. As such the estimated values of these health parameters are no longer representative of the physical health parameters they are based on. In particular the tenth health parameter, LPT flow capacity, exhibits an extremely large difference between its actual value and its tuned value resulting in a percent squared error of 135.59 in this health parameter alone. The three health parameters that are not estimated, (i.e., $\eta_{\mathrm{FAN}}, \gamma_{\mathrm{LPC}}$, and $\eta_{\mathrm{LPT}}$ ), are treated as if their estimated values are zero. Thus they all exhibit squared estimation errors of approximately 4.0, which is the variance of their respective values based upon $P_{h}$. Based on Table 6 it can also be seen that the reduced order tuner vectors, $q$, selected to minimize MAP health parameter estimation errors (vectors a, b, c, and d) result in Kalman filter SSEE's ranging from 17.55 to 38.28 , a 91 to 81 percent improvement over Kalman estimation of a subset of health parameters. This improvement is significant and highly encouraging. The $q$ vectors selected to minimize auxiliary parameter estimation errors (vectors $\mathrm{e}, \mathrm{f}, \mathrm{g}$, and $\mathrm{h}$ ) result in SSEE's ranging from 39.68 to 152.21 , an 80 to 24 percent improvement over Kalman estimation of a subset of health parameters. This too is a significant improvement, albeit not as large as that achieved by estimating vectors a through $\mathrm{d}$. However, this is not particularly surprising as tuners e through $\mathrm{h}$ are optimized for auxiliary parameter estimation, not health parameter estimation. An observation that is noteworthy is the relatively large variation in estimation accuracy provided by the different reduced order vectors. While these vectors produce relatively consistent health parameter estimation accuracy when applied to MAP estimation, their individual Kalman estimation SSEE's vary considerably.

TABLE 6.-KALMAN FILTER HEALTH PARAMETER EXPERIMENTAL PERCENT SQUARED ESTIMATION ERRORS

\begin{tabular}{|c|c|c|c|c|c|c|c|c|c|c|c|c|}
\hline $\begin{array}{c}\text { Estimated } \\
\text { parameters }\end{array}$ & $\begin{array}{l}\text { Tuner } \\
\text { vector }\end{array}$ & $\eta_{\text {FAN }}$ & $\gamma_{\mathrm{FAN}} *$ & $\eta_{L_{P C}^{*}}$ & $\gamma_{\text {LPC }}$ & $\eta_{\mathrm{HPC}^{*}}$ & $\gamma_{\mathrm{HPC}^{*}}$ & $\eta_{\mathrm{HPT}^{*}}$ & $\gamma_{\mathrm{HPT}^{*}}$ & $\eta_{\text {LPT }}$ & $\gamma_{\mathrm{LPT}^{*}}$ & $\operatorname{SSEE}(\hat{h})$ \\
\hline $\begin{array}{l}\text { Subset of } 7 \text { health } \\
\text { parameter tuners } \\
(\text { denoted by } *)\end{array}$ & N/A & 3.44 & 7.07 & 12.23 & 4.22 & 0.40 & 0.63 & 33.12 & 0.20 & 4.00 & 135.5 & 200.90 \\
\hline \multirow{4}{*}{$\begin{array}{l}\text { Tuner vector } \\
\text { selected to } \\
\text { minimize h error }\end{array}$} & $\mathrm{a}$ & 1.89 & 2.13 & 2.95 & 2.00 & 0.40 & 0.64 & 1.13 & 0.20 & 2.41 & 3.80 & 17.55 \\
\hline & $\mathrm{b}$ & 5.54 & 6.20 & 4.76 & 6.28 & 0.39 & 0.44 & 2.01 & 0.19 & 5.00 & 7.47 & 38.28 \\
\hline & $\mathrm{c}$ & 1.98 & 2.14 & 6.31 & 1.97 & 0.39 & 0.53 & 2.74 & 0.20 & 2.37 & 10.37 & 29.01 \\
\hline & $\mathrm{d}$ & 1.82 & 1.86 & 2.49 & 1.74 & 0.39 & 0.53 & 2.48 & 0.20 & 4.21 & 9.41 & 25.11 \\
\hline \multirow{4}{*}{$\begin{array}{l}\text { Tuner vector } \\
\text { selected to } \\
\text { minimize z error }\end{array}$} & e & 5.07 & 6.16 & 13.66 & 3.47 & 0.31 & 0.45 & 1.12 & 0.19 & 15.89 & 3.81 & 50.11 \\
\hline & $\mathrm{f}$ & 20.16 & 42.28 & 18.00 & 37.45 & 0.29 & 0.32 & 2.47 & 0.19 & 21.41 & 9.65 & 152.21 \\
\hline & $\mathrm{g}$ & 5.24 & 45.43 & 3.34 & 35.14 & 0.31 & 0.56 & 4.31 & 0.20 & 30.74 & 17.29 & 142.56 \\
\hline & $\mathrm{h}$ & 2.88 & 5.91 & 6.49 & 3.74 & 0.37 & 0.53 & 1.56 & 0.18 & 11.47 & 6.55 & 39.68 \\
\hline
\end{tabular}

Table 7 shows the experimental auxiliary parameter estimation accuracy results obtained when implementing the Kalman filter using the $q$ vectors. Applying the conventional Kalman filter approach of estimating a subset of health parameters results in an auxiliary parameter SSEE of 656.37. Applying Kalman filter estimation of the $q$ vectors optimally selected for MAP health parameter estimation (vectors $\mathrm{a}, \mathrm{b}, \mathrm{c}$, and d) results in auxiliary parameter SSEE's ranging from 205.61 to 263.16, a 69 to 60 percent improvement over Kalman estimation of a subset of health parameter tuners. This is an encouraging finding, and demonstrates that a tuner vector can be applied which will significantly improve auxiliary parameter estimation accuracy. Applying Kalman filter estimation of the $q$ vectors optimally selected for auxiliary parameter MAP estimation (vectors $\mathrm{e}, \mathrm{f}, \mathrm{g}$, and $\mathrm{h}$ ) results in auxiliary parameter SSEE's ranging from 678.70 to 2114.59 . This is actually 3 to 222 percent worse than simply choosing a subset of health parameters to serve as tuners. It is noted that these results are significantly worse than those obtained when applying vectors $\mathrm{a}, \mathrm{b}, \mathrm{c}$, and $\mathrm{d}$. These results reveal that estimating a reduced order $q$ vector optimal for MAP estimation of auxiliary parameters does not guarantee good results when using a Kalman filter. In fact, this example shows that the Kalman filter auxiliary parameter estimation accuracy provided by these tuners is rather poor. 
TABLE 7.-KALMAN FILTER AUXILIARY PARAMETER

EXPERIMENTAL SQUARED ESTIMATION ERRORS

\begin{tabular}{|l|c|c|c|c|c|c|}
\hline \multicolumn{1}{|c|}{$\begin{array}{c}\text { Estimated } \\
\text { parameters }\end{array}$} & $\begin{array}{c}\text { tuner } \\
\text { vector }\end{array}$ & $\begin{array}{c}\mathrm{T} 40 \\
\left({ }^{\circ} \mathrm{R}\right)\end{array}$ & $\begin{array}{c}\mathrm{T} 50 \\
\left({ }^{\circ} \mathrm{R}\right)\end{array}$ & $\begin{array}{c}\text { Fn } \\
(\%)\end{array}$ & $\begin{array}{c}\text { SmLPC } \\
(\%)\end{array}$ & $\operatorname{SSEE}(\hat{z})$ \\
\hline $\begin{array}{l}\text { Subset of 7 health } \\
\text { parameter tuners }\end{array}$ & N/A & 74.68 & 573.52 & 4.40 & 3.77 & $\mathbf{6 5 6 . 3 7}$ \\
\hline & $\mathrm{a}$ & 77.12 & 125.81 & 1.00 & 1.68 & $\mathbf{2 0 5 . 6 1}$ \\
$\begin{array}{l}\text { Tuner vector selected } \\
\text { to minimize h error }\end{array}$ & $\mathrm{b}$ & 74.64 & 147.82 & 2.39 & 4.48 & $\mathbf{2 2 9 . 3 2}$ \\
& $\mathrm{c}$ & 77.64 & 182.70 & 1.10 & 1.72 & $\mathbf{2 6 3 . 1 6}$ \\
& $\mathrm{d}$ & 76.14 & 131.33 & 0.88 & 1.55 & $\mathbf{2 0 9 . 9 0}$ \\
\hline & $\mathrm{e}$ & 75.61 & 800.83 & 2.94 & 3.47 & $\mathbf{8 8 2 . 8 6}$ \\
Tuner vector selected & $\mathrm{f}$ & 74.16 & 1221.61 & 17.79 & 27.05 & $\mathbf{1 3 4 0 . 6 2}$ \\
& $\mathrm{g}$ & 74.48 & 1992.54 & 20.23 & 27.34 & $\mathbf{2 1 1 4 . 5 9}$ \\
& $\mathrm{h}$ & 73.34 & 599.05 & 2.78 & 3.53 & $\mathbf{6 7 8 . 7 0}$ \\
\hline
\end{tabular}

\section{Discussion}

The process of selecting and evaluating the reduced order tuner vectors has revealed several significant findings. For MAP estimation applications, experimentally obtained mean squared estimation errors were found to be in agreement with theoretical predictions. This finding, combined with the fact that the MAP estimation accuracy of the reduced order tuner vectors approaches that obtained when estimating the entire health parameter vector verifies that the optimal iterative search routine is indeed performing as designed - it is returning a tuning vector optimal for MAP estimation. However, the ultimate intent is to apply these tuners to Kalman filter-based estimation. Implementation of the reduced order tuner vectors in a Kalman filter demonstrated that improved estimation accuracy, relative to the conventional approach of selecting a subset of health parameters to serve as tuners, could be obtained. However, this did not hold true in all cases. Specifically, the reduced order $q$ vectors selected for MAP auxiliary parameter estimation resulted in degraded Kalman estimation performance. Furthermore, while there was not a significant difference in the estimation accuracy yielded by the individual tuners when used in MAP estimation, there was considerable difference between them when used in Kalman estimation. In summary, it has been shown that appropriately selected reduced order tuners can improve Kalman estimation accuracy over using a subset of health parameters, but tuners optimal for MAP estimation are not necessarily optimal for Kalman filter estimation applications. Based upon these findings, follow on work is being pursued to develop a methodology that will systematically select a reduced order tuner vector optimal for Kalman filter applications. This work uses an approach similar to the one presented in this paper for MAP estimator tuner selection. Namely, theoretical mean squared estimation error equations, consisting of squared bias and variance, are derived for the Kalman filter and applied within an iterative search routine designed to return an optimal reduced order tuner vector. Initial results have shown that reduced order tuner vectors can be found that consistently result in near optimal Kalman filter estimation accuracy. Furthermore, the Kalman filter estimation accuracy obtained by implementing these reduced order tuner vectors significantly exceeds that obtained by implementing the MAP-based reduced order tuner vectors as presented in this paper. The full results of this study will be presented in an upcoming conference paper to be published by the authors (Ref. 11).

\section{Conclusions}

A systematic approach to reduced order model tuning parameter selection for online engine performance estimation has been presented. This technique is specifically focused on the underdetermined aircraft engine parameter estimation problem where there are fewer sensor 
measurements than unknown health parameters that impact engine outputs. The technique creates and applies a linear transformation matrix, $V^{*}$, to select a reduced order vector of tuning parameters that are a linear combination of all health parameters, and of low enough dimension to be estimated by a Kalman filter. In order to facilitate reduced order tuner parameter selection, a multi-parameter iterative search was applied to optimally select $V^{*}$ to minimize the theoretical mean squared error of a maximum a posteriori (MAP) estimator. Results have shown that while the transformation matrix returned by the optimization routine is not unique, the routine is effective in returning a transformation matrix that is near optimal for MAP estimation applications regardless of its initial starting guess of the matrix. This was theoretically predicted and experimentally validated through Monte Carlo simulation studies. It was also demonstrated that a reduced order tuner vector could be generated that performs reasonably well for the dual objective of minimizing both health and auxiliary parameter estimation errors. The estimation accuracy achieved when applying the reduced order tuner vectors for their intended application, Kalman filter-based estimation, was also evaluated experimentally. These studies revealed that the application of a reduced order tuner vector can yield significant improvements in Kalman filter estimation accuracy relative to the conventional approach of selecting a subset of health parameters to serve as tuners. However, there is no guarantee that a vector of reduced order tuners optimal for MAP estimation will be optimal when used for Kalman filter estimation. Based on these results it is concluded that the appropriate approach is to revise the methodology to perform the systematic selection of reduced order tuner parameters specifically for Kalman filter-based estimation applications. Follow on work towards that goal is currently being pursued.

\section{References}

1. Luppold, R.H. Roman, J.R., Gallops, G.W., and Kerr, L.J., "Estimating In-Flight Engine Performance Variations Using Kalman Filter Concepts," AIAA-89-2584, AIAA 25th Joint Propulsion Conference, Monterey, CA, July 10-13, 1989.

2. Volponi, A., "Enhanced Self-Tuning On-Board Real-Time Model (eSTORM) for Aircraft Engine Performance Health Tracking," NASA/CR—2008-215272, Jul. 2008.

3. Kumar, A., and Viassolo, D., "Model-Based Fault Tolerant Control," NASA/CR—2008-215273, Sep. 2008.

4. España, M.D., "Sensor Biases Effect on the Estimation Algorithm for Performance-Seeking Controllers," J. of Propulsion and Power, Vol. 10, No. 4, 1994, pp. 527-532.

5. Litt, J.S., "An Optimal Orthogonal Decomposition Method for Kalman Filter-Based Turbofan Engine Thrust Estimation," J. of Engineering for Gas Turbines and Power, Vol. 130/011601-1, Jan. 2008.

6. Doel, D.L., "An Assessment of Weighted-Least-Squares-Based Gas Path Analysis," J. of Engineering for Gas Turbines and Power, Vol. 116, pp. 336-373, April 1994.

7. Volponi, A.J., et al, "Gas Turbine Condition Monitoring and Fault Diagnostics", Von Karman Institute for Fluid Dynamics, Lecture Series 2003-01, Jan. 2003.

8. Simon, D., Optimal State Estimation, Kalman, $H \infty$, and Nonlinear Approaches, John Wiley \& Sons, Inc., Hoboken, NJ, 2006.

9. Gelb, A., Applied Optimal Estimation, MIT Press, Cambridge, MA, 1974, pp. 104-105.

10. Frederick, D.K., DeCastro, J.A., and Litt, J.S., "User's Guide for the Commercial Modular AeroPropulsion System Simulation (C-MAPSS)," NASA/TM-2007-215026, Oct. 2007.

11. Simon, D.L., and Garg, S., "Optimal Tuner Selection for Kalman Filter-Based Aircraft Engine Performance Estimation," ASME Turbo Expo Conference, paper GT2009-59684, ASME, Orlando, FL, Jun. 8-12, 2009 (to be published). 


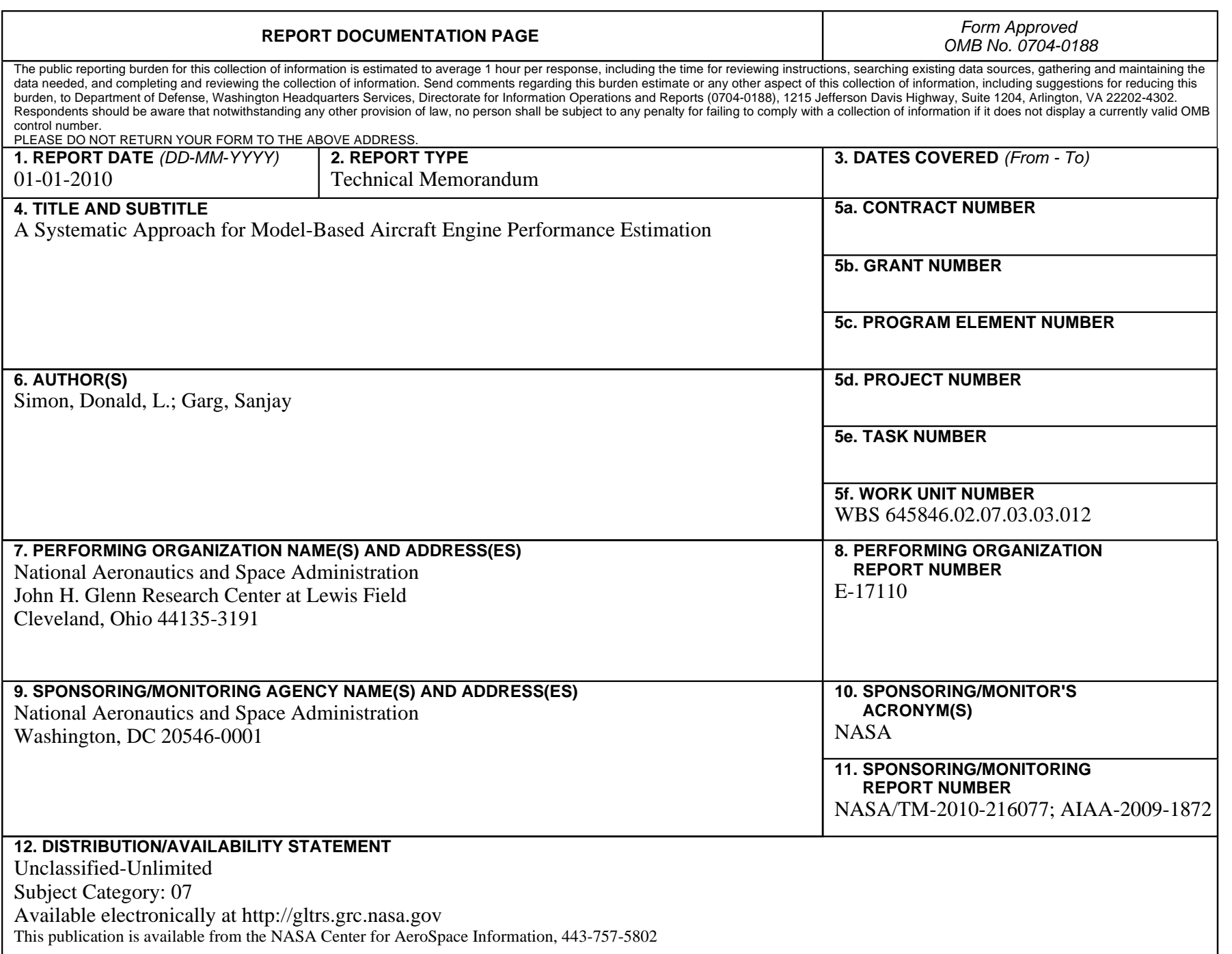

\section{SUPPLEMENTARY NOTES}

\section{ABSTRACT}

A requirement for effective aircraft engine performance estimation is the ability to account for engine degradation, generally described in terms of unmeasurable health parameters such as efficiencies and flow capacities related to each major engine module. This paper presents a linear point design methodology for minimizing the degradation-induced error in model-based aircraft engine performance estimation applications. The technique specifically focuses on the underdetermined estimation problem, where there are more unknown health parameters than available sensor measurements. A condition for Kalman filter-based estimation is that the number of health parameters estimated cannot exceed the number of sensed measurements. In this paper, the estimated health parameter vector will be replaced by a reduced order tuner vector whose dimension is equivalent to the sensed measurement vector. The reduced order tuner vector is systematically selected to minimize the theoretical mean squared estimation error of a maximum a posteriori estimator formulation. This paper derives theoretical estimation errors at steady-state operating conditions, and presents the tuner selection routine applied to minimize these values. Results from the application of the technique to an aircraft engine simulation are presented and compared to the estimation accuracy achieved through conventional maximum a posteriori and Kalman filter estimation approaches. Maximum a posteriori estimation results demonstrate that reduced order tuning parameter vectors can be found that approximate the accuracy of estimating all health parameters directly. Kalman filter estimation results based on the same reduced order tuning parameter vectors demonstrate that significantly improved estimation accuracy can be achieved over the conventional approach of selecting a subset of health parameters to serve as the tuner vector. However, additional development is necessary to fully extend the methodology to Kalman filter-based estimation applications.

\section{SUBJECT TERMS}

Aircraft engines; Systems health monitoring; Gas turbine engines; Kalman filtering; State estimation

\begin{tabular}{|c|c|c|c|c|c|}
\hline \multicolumn{3}{|c|}{ 16. SECURITY CLASSIFICATION OF: } & \multirow{2}{*}{$\begin{array}{l}\text { 17. LIMITATION OF } \\
\text { ABSTRACT } \\
\text { UU }\end{array}$} & \multirow{2}{*}{$\begin{array}{l}\text { 18. NUMBER } \\
\text { OF } \\
\text { PAGES } \\
25\end{array}$} & \multirow{2}{*}{$\begin{array}{l}\text { 19a. NAME OF RESPONSIBLE PERSON } \\
\text { STI Help Desk (email:help@sti.nasa.gov) } \\
\text { 19b. TELEPHONE NUMBER (include area code) } \\
\text { 443-757-5802 }\end{array}$} \\
\hline $\begin{array}{l}\text { a. REPORT } \\
U\end{array}$ & $\begin{array}{l}\text { b. ABSTRACT } \\
\text { U }\end{array}$ & $\begin{array}{l}\text { c. THIS } \\
\text { PAGE } \\
\text { U }\end{array}$ & & & \\
\hline
\end{tabular}



